The Astronomical Journal, 133:1927-1933, 2007 May

(C) 2007. The American Astronomical Society. All rights reserved. Printed in U.S.A.

\title{
INFRARED EMISSION FROM THE DUSTY DISK ORBITING GD 362, AN EXTERNALLY POLLUTED WHITE DWARF
}

\author{
M. Jura, ${ }^{1}$ J. FArihi, ${ }^{2}$ B. Zuckerman, ${ }^{1}$ And E. E. BecKlin ${ }^{1}$ \\ Received 2006 October 31; accepted 2007 January 14
}

\begin{abstract}
We report Spitzer Space Telescope photometry between 3.6 and $24 \mu \mathrm{m}$ and spectroscopy between 5 and $15 \mu \mathrm{m}$ of GD 362, a white dwarf with an effective temperature near 10,000 K that displays a remarkably high concentration of metals in its photosphere and a thermal infrared excess. We approximately reproduce both the infrared continuum and the very strong $10 \mu \mathrm{m}$ silicate emission feature with a model of an orbiting dusty disk that is flat out to 50 stellar radii and warped between 50 and 70 stellar radii. The relatively small amount of cold material implied by the weak $24 \mu \mathrm{m}$ flux argues that the disk lies within the Roche radius of the star, and we may be witnessing a system where an asteroidal-size body has been tidally destroyed. If so, determination of the photospheric metal abundances may measure the bulk composition of an extrasolar minor planet.
\end{abstract}

Key words: circumstellar matter — minor planets, asteroids — white dwarfs

\section{INTRODUCTION}

Although little is known about the fate of a planetary system when a main-sequence star eventually becomes a white dwarf, we can now detect with modern instrumentation indirect signatures of disrupted comets and asteroids in the atmospheres and circumstellar environments of cool white dwarfs. Here we report infrared observations of GD 362 that strengthen the argument that this star is orbited by debris from a tidally disrupted asteroidalsize body.

Gravitational settling of elements heavier than the dominant light gas - either hydrogen or helium - through the relatively thin outer convective zones of white dwarfs occurs rapidly (Paquette et al. 1986). Therefore, the atmospheres of the stars that are cool enough that radiative levitation is unimportant are anticipated to be metal-free. In agreement with this expectation, $\sim 75 \%$ of hydrogen-rich white dwarfs with effective temperatures less than $10,000 \mathrm{~K}$ have calcium abundances below $10^{-5}$ solar (Zuckerman et al. 2003). However, some cool white dwarfs do display atmospheric metals, and these stars are almost certainly externally polluted. One of the most remarkable metal-bearing cool white dwarfs is GD 362, which has approximately solar abundances of calcium, magnesium, and iron (Gianninas et al. 2004). Motivated by this striking composition, Becklin et al. (2005) and Kilic et al. (2005) independently reported groundbased observations showing that GD 362 has an infrared excess produced by circumstellar dust that is likely to be the source of the external pollution required to explain the star's photospheric composition. After G29-38 (Zuckerman \& Becklin 1987), GD 362 is the second single white dwarf discovered to display an infrared excess. Here we report Spitzer Space Telescope (Werner et al. 2004) observations of GD 362 to study better its infrared emission and to constrain models of the origin of the circumstellar dust.

A promising model to explain both the infrared excesses and the relatively high metal abundances within both GD 362 and

\footnotetext{
${ }^{1}$ Department of Physics and Astronomy and Center for Astrobiology, University of California, Los Angeles, CA 90095-1562,USA; jura@astro.ucla.edu,ben@ astro.ucla.edu, becklin@astro.ucla.edu.

${ }^{2}$ Gemini Observatory, 670 North A'ohoku Place, Hilo, HI 96720, USA; jfarihi@gemini.edu.
}

G29-38 is that these stars accrete from an opaque dust disk, reminiscent of Saturn's rings, produced by the tidal disruption of an asteroid (Jura 2003; Becklin et al. 2005). A somewhat different approach was taken by Reach et al. (2005a), who proposed that the infrared data for G29-38 can be explained by an optically thin cloud. These two different models of the infrared excesses around white dwarfs can imply dramatically different amounts of mass in the circumstellar environment. If there is an opaque disk, there may be in excess of $\sim 10^{24} \mathrm{~g}$ of circumstellar dust, comparable to the mass of Ceres, the largest asteroid in the solar system. If the disk is optically thin, there may be only $\sim 10^{18} \mathrm{~g}$ of circumstellar dust, comparable to the mass of a comet, although there could be a more massive population of large unseen bodies. In $\S 2$ we present the observations while in $\S 3$ we describe our model. In $\S 4$ we discuss our results and in $\S 5$ we present our conclusions.

\section{OBSERVATIONS}

Infrared Array Camera (IRAC; Fazio et al. 2004) observations of GD 362 were executed 2005 August 25 at 3.6, 4.5, 5.7, and $7.9 \mu \mathrm{m}$. A 20 point cycling dither pattern was used with medium-scale steps: five frames of $12 \mathrm{~s}$ taken at each point in the dither pattern, yielding a total on-source time of $1200 \mathrm{~s}$ in all four filters. At each of the four wavelengths, individual frames were combined into a single reduced image via the IRAC calibration pipeline, version 12.4. Aperture photometry was performed on the target in the combined image using standard IRAF tasks. Both the flux and signal-to-noise ratio $(\mathrm{S} / \mathrm{N})$ were measured in a 3 pixel $\left(3.6^{\prime \prime}\right)$ radius, with a sky annulus of 10-20 pixels. This measured flux was then extrapolated to the IRAC standard 10 pixel aperture using appropriate corrections (IRAC Data Handbook, ver. 3.0, 2006). For all four channels, the $\mathrm{S} / \mathrm{N}$ was sufficiently high $(>25-100)$ that the photometric errors are dominated by the $10 \%$ absolute calibration uncertainty of the IRAC instrument that results from the as-yet to be fully characterized responses of the filter bandpasses (Quijada et al. 2004; Reach et al. 2005b; Hines et al. 2006). The results are listed in Table 1; no color corrections have been applied.

GD 362 was imaged with the Multiband Imaging Photometer for Spitzer (MIPS; Rieke et al. 2004) in the $24 \mu \mathrm{m}$ filter. The observations consisted of 20 cycles with the small field of view, the default 14 point dither pattern, and $10 \mathrm{~s}$ individual exposures for 
TABLE 1

Spitzer Photometry of GD 362

\begin{tabular}{|c|c|}
\hline $\begin{array}{l}\text { Wavelength } \\
\qquad(\mu \mathrm{m})\end{array}$ & $\begin{array}{l}\text { Flux } \\
(\mathrm{mJy})\end{array}$ \\
\hline $3.6 \ldots \ldots \ldots$ & $0.380 \pm 0.038$ \\
\hline 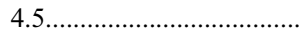 & $0.395 \pm 0.040$ \\
\hline 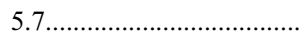 & $0.425 \pm 0.043$ \\
\hline . & $0.644 \pm 0.064$ \\
\hline 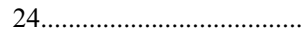 & $0.22 \pm 0.06$ \\
\hline
\end{tabular}

a total of $2800 \mathrm{~s}$ of on-source integration. The data were processed through the MIPS calibration pipeline, version 12.4, to create a single combined and reduced image. Standard IRAF aperture photometry was performed with a $6^{\prime \prime}$ radius ( 2.35 pixels) utilizing appropriate sky annuli and aperture corrections as determined by the MIPS instrument team and instrument support team. ${ }^{3}$ The measurement yields a $\mathrm{S} / \mathrm{N}$ of 4.3 and the total error is the inverse of the $\mathrm{S} / \mathrm{N}$ summed in quadrature with the $10 \%$ absolute calibration error of the $24 \mu \mathrm{m}$ camera (MIPS Data Handbook, ver. $3.2,2006$ ). The result is listed in Table 1 . From previous ground-based data (Becklin et al. 2005) and from inspection of the Spitzer IRAC and MIPS images, contamination by other sources of the fluxes we report for GD 362 does not appear to be a problem.

Spectroscopy over the 5-15 $\mu \mathrm{m}$ region of GD 362 was performed with the Infrared Spectrograph (IRS; Houck et al. 2004) on 2006 April 18. The instrument was operated in staring mode at two positions, both along the Short-Low 1 module slit (spectral range between 7.4 and $14.5 \mu \mathrm{m}$ and spectral resolution $\lambda / \Delta \lambda$ between 64 and 128) and along the Short-Low 2 module slit (spectral range between 5.2 and $7.7 \mu \mathrm{m}$ and spectral resolution $\lambda / \Delta \lambda$ between 80 and 128), with $240 \mathrm{~s}$ exposures executed at each position, repeated twice for a total on-source time of $960 \mathrm{~s}$ in each module. The data were processed through the IRS calibration pipeline, version 14.0, to create reduced and combined exposures at each nod position in each module. These combined nodded frames were subtracted from one another to eliminate sky signal and were processed with the data reduction program SPICE to perform spectral extraction. The extracted spectra in each ShortLow module were averaged to a single spectrum to increase the $\mathrm{S} / \mathrm{N}$. The extractions were performed twice: first with the default aperture function (8 pixels at $12 \mu \mathrm{m}$ ) in order to assess the proper calibration level, which does not exist for custom (nondefault) aperture extractions; and second with a smaller aperture function (4 pixels at $12 \mu \mathrm{m}$ ), which yielded a higher $\mathrm{S} / \mathrm{N}$ extraction. The custom extraction was scaled appropriately to match the overall level of the default extraction and all the orders were combined (and averaged in regions of overlap, including the Short-Low 1 bonus section, which has a spectral range of 7.3-8.7 $\mu \mathrm{m}$ ). In our data we clearly detect only silicate emission; higher $\mathrm{S} / \mathrm{N}$ is required to determine whether other features are present. The spectrum and the associated errors are displayed in Figure 1.

The Spitzer data show a strong silicate feature near $10 \mu \mathrm{m}$, consistent with the ground-based photometry presented by Becklin et al. (2005). The profile of the silicate emission from GD 362 resembles the emission feature seen by Reach et al. (2005a) for G29-38, and it is distinctly different from the profile of interstellar silicates (Kemper et al. 2004) also shown in Figure 1. If the dust around GD 362 has an asteroidal origin, its spectrum may re-

\footnotetext{
${ }^{3}$ See http://ssc.spitzer.caltech.edu/mips/apercorr.
}

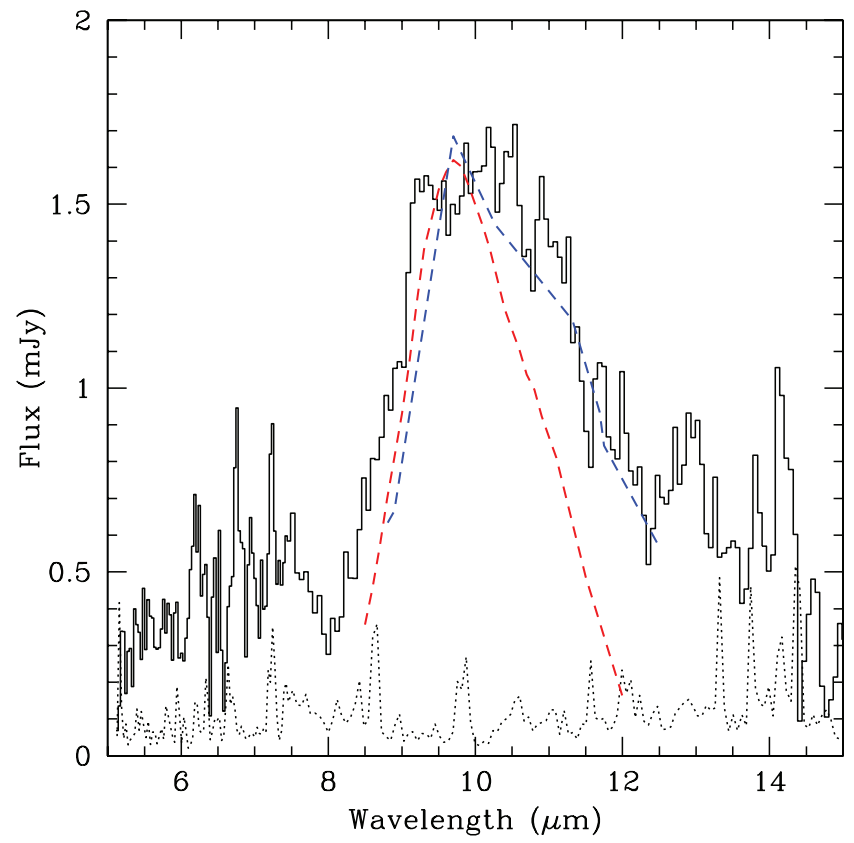

FIG. 1.-IRS spectrum of GD 362. The errors are shown as the dotted black line; the feature near $14 \mu \mathrm{m}$ is a detector/instrument artifact. For comparison, we display the scaled profiles of interstellar silicates (Kemper et al. 2004) as a dashed red line and the emission from BD +20 307 (Song et al. 2005) as a dashed blue line.

semble that of warm $(T>200 \mathrm{~K})$ dust around main-sequence stars (see Chen et al. 2006). BD +20 307 notably exhibits a strong silicate feature from grains at a temperature near $650 \mathrm{~K}$ (Song et al. 2005), and we see from Figure 1 that the spectra of GD 362 and BD +20 307 agree fairly well, except that the blue wing of the feature is stronger in GD 362, which as described below can be understood if the grain temperature is near $\sim 1100 \mathrm{~K}$.

\section{PROPOSED MODEL}

Becklin et al. (2005) found that their ground-based continuum data from 1.2 to $11.3 \mu \mathrm{m}$ for GD 362 could be moderately well matched with a geometrically thin, flat, opaque disk whose total thickness is much less than the diameter of the star and where the dust temperature is a function only of the radial distance from the star. The flux in the $N^{\prime}(11.3 \mu \mathrm{m})$ filter in the MICHELLE instrument on the Gemini North telescope was substantially above the prediction of the model disk. This discrepancy is now understood as a result of the very strong silicate emission (see Fig. 1) entering into the $N^{\prime}$ filter, which has a full width half-maximum of $2.4 \mu \mathrm{m}$. Without any temperature gradient vertical to the disk, this model cannot, by itself, explain the strong silicate emission. Therefore, we present a more sophisticated disk model.

We assume a passive disk with three regions so that at every radius, the disk reradiates the flux incident upon it. A schematic edge-on view of our model is shown in Figure 2. The inner region has sufficient surface density that it is opaque at all wavelengths of interest. Furthermore, thermal conductivity is sufficiently great in this region that the disk is vertically isothermal. Further out, the disk is still opaque but thermal conductivity is less important, and there is a temperature gradient in the disk. The outer region of the disk is thick enough to absorb most of the incident radiation, but it is optically thin in the infrared. This outermost portion of the disk has relatively little mass, and therefore it can be warped. Our model is somewhat artificial since the three regions are distinct, and therefore it has unrealistic temperature jumps at the boundaries 


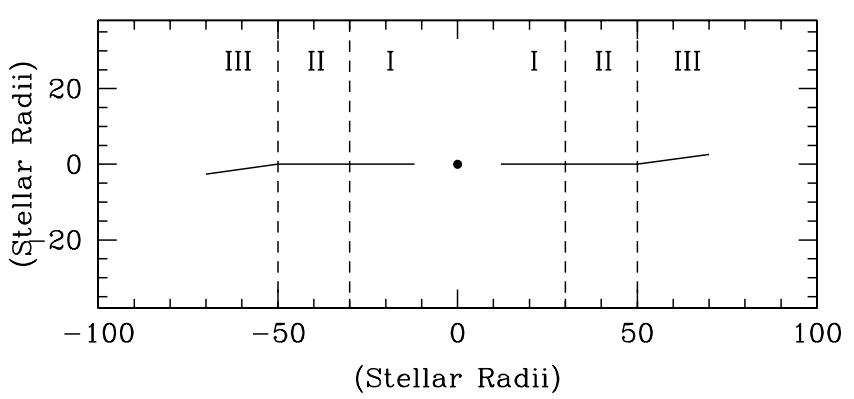

FIG. 2.-Schematic diagram of an edge-on view of our model for the disk orbiting GD 362. The star is denoted by the small point (approximately to scale) at the origin, while the different zones of the disk are labeled. The azimuthal variation of the warping is not known; here we just illustrate one possibility.

between the regions. These temperature jumps are most important in the midplane of the disk, which emits little light, and thus the model predictions are not strongly affected by our overly simplified description of the disk temperature variations.

If $R$ denotes the distance from the star and $\tau_{\nu}$ denotes the optical depth from the top of the disk measured downward, then as with a stellar atmosphere, the emergent intensity $I_{\nu}$ for a disk seen at inclination angle $i$, defined such that a face-on disk has $i=0^{\circ}$, is

$$
I_{\nu}(R)=\int_{0}^{\infty} B_{\nu}\left(T\left(\tau_{\nu}\right)\right) \exp \left(-\tau_{\nu} / \cos i\right)\left(d \tau_{\nu} / \cos i\right) .
$$

If $D_{*}$ denotes the distance to GD 362, the flux received at Earth, $F_{\nu}$, is

$$
F_{\nu}=D_{*}^{-2} \cos i \int_{R_{\mathrm{in}}}^{R_{\mathrm{out}}} 2 \pi R I_{\nu}(R) d R
$$

To model the observed flux, we compute the temperature at all locations within the disk.

\subsection{Region I: The Inner Opaque, Vertically Isothermal Region}

Close to the star, unshielded grains can get very hot and rapidly sublimate. However, if thermal conductivity between a grain and the surrounding gas is important in regulating a grain's temperature, then the disk can be approximately vertically isothermal. Consequently, even close to the star, grains at the top of the disk can survive.

The importance of thermal conduction depends on the amount of gas in the disk. In the solar system, Ceres has a mean density of $2.1 \mathrm{~g} \mathrm{~cm}^{-3}$ and an estimated water content between $17 \%$ and $27 \%$ by mass (McCord \& Sotin 2005; Thomas et al. 2005). The tidal destruction of an analog to Ceres could thus provide the disk with gaseous hydrogen, and a gas-to-dust ratio by mass between 0.02 and 0.03 . Additional elements such as sodium and potassium can be gaseous at $1300 \mathrm{~K}$ in metal-enhanced environments (Ebel \& Grossman 2000), but we ignore this contribution to the gas composition.

Thermal conduction is important in determining the grain temperature $T$ when the rates of energy exchange from the grain surface to its surroundings by gas particles and photons are comparable. This situation occurs when (Burke \& Hollenbach 1983)

$$
n_{0} k_{\mathrm{B}} T\left(\frac{8 k_{\mathrm{B}} T}{\pi m}\right)^{1 / 2} \approx \sigma_{\mathrm{SB}} T^{4},
$$

where $\sigma_{\mathrm{SB}}$ is the Stephan-Boltzmann constant, $k_{\mathrm{B}}$ is Boltzmann's constant, $n_{0}$ is the gas number density in the midplane of the disk, and $m$ is the mean atomic weight of the gas. If $\Sigma_{\text {gas }}$ denotes the mass surface density of the gaseous fraction of the disk, with the usual assumption that the disk is in vertical hydrostatic equilibrium, then at distance $R$ from a star of mass $M_{*}$,

$$
n_{0}=\frac{\Sigma_{\mathrm{gas}}}{m}\left(\frac{G M_{*} m}{2 \pi R^{3} k_{\mathrm{B}} T}\right)^{1 / 2} .
$$

From equations (3) and (4), thermal conduction is important if

$$
\Sigma_{\mathrm{gas}} \geq \frac{\pi m \sigma_{\mathrm{SB}} T^{3}}{2 k_{\mathrm{B}}}\left(\frac{R^{3}}{G M_{*}}\right)^{1 / 2} .
$$

Adopting very approximate values of $R \sim 10^{10} \mathrm{~cm}, T \sim 1000 \mathrm{~K}$, $M_{*} \sim 0.8 M_{\odot}$, and $m=3.3 \times 10^{-24} \mathrm{~g}$ (for $\mathrm{H}_{2}$ ), thermal conduction is important where $\Sigma_{\text {gas }} \geq 0.2 \mathrm{~g} \mathrm{~cm}^{-2}$. If the disk has a mass of $10^{24} \mathrm{~g}$ and a mean radius of $\sim 10^{10} \mathrm{~cm}$ (Becklin et al. 2005), the average dust surface density is $3000 \mathrm{~g} \mathrm{~cm}^{-2}$. Therefore, in regions where the gas density is $\gg 10^{-4}$ of the grain density as expected from the disruption of an analog to Ceres (see above), thermal conduction is important. For a flat disk where the thermal conduction inhibits any vertical thermal gradient, the temperature is (see, for example, Chiang \& Goldreich 1997)

$$
T=\left(\frac{2}{3 \pi}\right)^{1 / 4}\left(\frac{R_{*}}{R}\right)^{3 / 4} T_{*} .
$$

\subsection{Region II: The Opaque Region with Vertical Temperature Gradients}

Beyond the innermost region, grains at the top of the disk can survive even without efficient thermal conduction, and there may be a vertical temperature gradient. To compute the grain temperature as a function of location, we adopt a model similar to that of a planetary atmosphere (Goody \& Yung 1989) or a disk around a pre-main-sequence star (Calvet et al. 1991). We define $\alpha$ as the ratio of the Planck mean opacities in the optical, $\bar{\chi}_{V}$, and infrared, $\bar{\chi}_{\mathrm{IR}}$ :

$$
\alpha=\frac{\bar{\chi}_{V}}{\bar{\chi}_{\mathrm{IR}}} .
$$

In this approach, we assume that the grains are heated by optical light and reradiate infrared light. We assume that all of the dust particles at a given distance from GD 362 have the same radius $a$, and therefore that at each location a single temperature characterizes the material. We also assume that the spectral character of the incident stellar radiation does not vary with optical depth so that $\bar{\chi}_{V}$ is constant throughout the atmosphere. We further neglect the temperature variations in $\bar{\chi}_{\mathrm{IR}}$. Consequently, $\alpha$ depends only on the grain size, and it varies as a function of its distance from the star since we allow for different-size grains at different radial locations. Below, in $\S 3.4$, we describe in more detail our sources for deriving $\alpha$.

If $\mu_{0}$ denotes the mean cosine of the average incidence angle of the starlight with respect to the normal to the disk and if we ignore limb darkening, then at distance $R$ from the star

$$
\mu_{0}=\frac{4}{3 \pi} \frac{R_{*}}{R}
$$


where $R_{*}$ denotes the radius of the star. Integrated over all frequencies, the mean intensity of the incident optical light, $J_{\text {opt }}(0)$, at the top of the disk is

$$
J_{\mathrm{opt}}(0)=\frac{R_{*}^{2}}{8 R^{2}} \frac{\sigma_{\mathrm{SB}} T_{*}^{4}}{\pi} .
$$

For convenience, we define $J^{\prime}(0)$ as

$$
J^{\prime}(0)=\frac{1}{8}\left(\frac{R_{*}}{R}\right)^{2}
$$

To find the grain temperature as a function of depth, we compute the mean intensity of the optical light within the atmosphere, $J_{\text {opt }}(\tau)$, from

$$
J_{\text {opt }}(\tau)=J^{\prime}(0)\left(\frac{\sigma_{\mathrm{SB}} T_{*}^{4}}{\pi}\right) \exp \left(-\frac{\alpha \tau}{\mu_{0}}\right) .
$$

Assuming that the infrared source function is $\left(\sigma_{\mathrm{SB}} T^{4} / \pi\right)$, an approximate solution to the equation of transfer for the temperature as a function of depth is (see Goody \& Yung 1989)

$$
\left(\frac{T}{T_{*}}\right)^{4}=J^{\prime}(0)\left[\left(\alpha-\frac{3 \mu_{0}^{2}}{\alpha}\right) \exp \left(-\frac{\alpha \tau}{\mu_{0}}\right)+\left(2 \mu_{0}+\frac{3 \mu_{0}^{2}}{\alpha}\right)\right] .
$$

Since we expect that $\alpha>1$ and $\mu_{0} \ll 1$, then at the "top" of the atmosphere where $\tau=0$,

$$
T(0) \approx\left(\frac{\alpha}{8}\right)^{1 / 4}\left(\frac{R_{*}}{R}\right)^{1 / 2} T_{*} .
$$

This result for $T(0)$ is that of a blackbody that is fully illuminated by the half of the star that is visible above the opaque disk. Deep in the disk where $\tau \gg 1$, we find from equations (8), (10), and (12) that the temperature asymptotically approaches the value

$$
T(\infty) \approx\left(\frac{1}{3 \pi}\right)^{1 / 4}\left(\frac{R_{*}}{R}\right)^{3 / 4} T_{*}
$$

This asymptotic temperature is 0.84 of the temperature predicted in the vertically isothermal disk, as given by equation (6).

\subsection{Region III: The Outer Optically Thin Region}

At a large enough distance from the star, the disk surface density is sufficiently small that the system is optically thin in the infrared. To compute the temperature in this region, we note that the disk may also be warped, as described in the Appendix. For simplicity, we define $\mu_{\text {warp }}$ as the mean warping angle, and analogous to equation (11), the mean intensity of optical light in the disk is

$$
J_{\mathrm{opt}}=J^{\prime}(0)\left(\frac{\sigma_{\mathrm{SB}} T_{*}^{4}}{\pi}\right) \exp \left(-\frac{\alpha \tau}{\mu_{\mathrm{warp}}}\right) .
$$

To compute the grain temperature, we use

\begin{tabular}{|c|c|c|c|c|c|c|c|}
\hline Region & $\begin{array}{l}R_{\min } \\
(R *)\end{array}$ & $\begin{array}{l}T_{\text {top }} \\
(\mathrm{K})\end{array}$ & $\begin{array}{l}T_{\text {mid }} \\
(\mathrm{K})\end{array}$ & $\begin{array}{c}R_{\max } \\
(R *)\end{array}$ & $\begin{array}{l}T_{\text {top }} \\
(\mathrm{K})\end{array}$ & $\begin{array}{l}T_{\text {mid }} \\
(\mathrm{K})\end{array}$ & $\alpha$ \\
\hline I ....................... & 12 & 1025 & 1025 & 30 & 520 & 520 & \\
\hline II ................... & 30 & 1170 & 430 & 50 & 910 & 260 & 1.5 \\
\hline III..................... & 50 & 1220 & 1030 & 70 & 1040 & 870 & 5 \\
\hline
\end{tabular}

$$
\frac{\sigma_{\mathrm{SB}} T^{4}}{\pi}=\alpha J_{\mathrm{opt}} \text {. }
$$

Assuming that $\mu_{\text {warp }} \ll 1$, we use a modified version of equation (1) to estimate the intensity from the warped portion by in-
TABLE 2

Model Parameters

tegrating to the optical depth where $J$ falls to $50 \%$ of its value at the top of the warped disk instead of to infinite optical depth. The azimuthal variation of the warp is not constrained by the data, only the mean warping angle.

\subsection{Results}

We now describe a specific model of the system to fit the data. Gianninas et al. (2004) derived a high gravity and mass for GD 362 with the assumption that the atmosphere is largely composed of hydrogen. A more recent study of the spectrum suggests that the Balmer line profiles are equally well reproduced if there is a significant amount of helium in the atmosphere of the star (Garcia-Berro et al. 2007). Independently, B. Zuckerman et al. (2007, in preparation) have detected an absorption line at $5876 \AA$ that is strong, direct evidence for the presence of helium. Consequently, the stellar parameters derived by Gianninas et al. (2004) need to be revised in the sense that their values for the inferred gravity and mass of the star are too large and their values for the inferred radius and distance to the star are too small. However, for the purposes of modeling the infrared emission from the star, we need only the effective temperature and the ratio of $R_{*} / D_{*}$. Here we adopt $R_{*} / D_{*}=5.2 \times 10^{-12}$, consistent with the distance and radius assumed by Becklin et al. (2005). In the infrared, the spectral shape of the star's photosphere is taken as a single-temperature blackbody and is independent of the distance to the star. Based on IRAC observations of other white dwarfs (Kilic et al. 2006), this approximation is usually accurate to better than the $10 \%$ measurement uncertainty in the fluxes for white dwarfs with effective temperatures warmer than $7000 \mathrm{~K}$ (Tremblay \& Bergeron 2007). Therefore, we follow Becklin et al. (2005) to separate the photospheric and circumstellar contributions to the infrared light. At $3.6 \mu \mathrm{m}$ the photosphere contributes about $30 \%$ of the total flux, and much less at longer wavelengths. In using equation (2) we assume a face-on disk.

Given that we have detected a silicate emission feature, we can constrain the size and composition of the dust particles. Reach et al. (2005a) fit the observations of G29-38 with a combination of amorphous silicate (to account for the peak of the feature) and forsterite (to explain its red wing). Here, for simplicity, to derive $\alpha$ we include only glassy silicate grains with an olivine stoichiometry described by Dorschner et al. (1995) for the case with equal abundances of magnesium and iron. As shown below, we find a satisfactory fit to the silicate feature without any forsterite because we include relatively large grains. Our model for the grains is similar to that used by Chen et al. (2006) to account for the silicate emission from the debris disk that emits near $360 \mathrm{~K}$ around the main-sequence star $\eta$ Crv.

We adopt model parameters to match the data; a summary of the dimensions and temperatures of our model disk is given in Table 2. Following Gianninas et al. (2004), we assume that the effective temperature of the star is $9740 \mathrm{~K}$, but this value may be revised upward perhaps as much as $\sim 10 \%$ with a better measure of the atmospheric helium abundance. We assume that the temperature of the grains is less than approximately $1200 \mathrm{~K}$ so that 


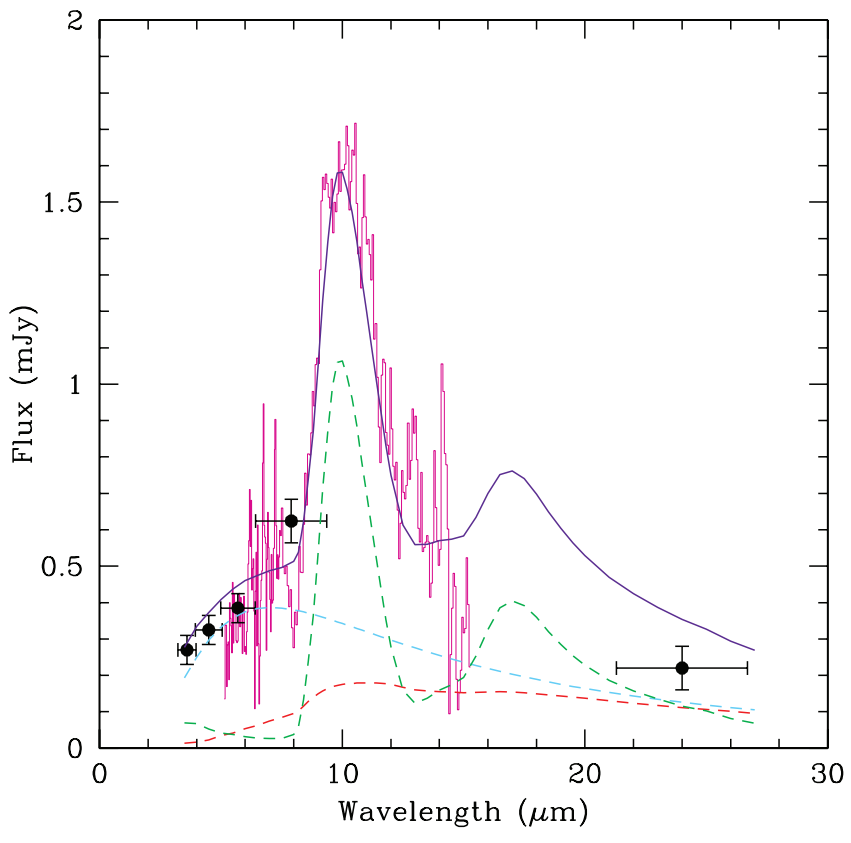

FIG. 3.-Comparison between photospheric-subtracted observations and predicted fluxes for the model disk described in the text. The points represent the IRAC and MIPS data listed in Table 1, while the IRS data from Fig. 1 are shown as the magenta line. The solid blue curve shows the total flux from the model, while the fluxes from regions I, II, and III are shown as dashed lines of cyan, red, and green, respectively.

they do not rapidly sublimate; this choice for the maximum grain temperature is consistent with the spectral energy distribution of the infrared excess near 3.6 $\mu \mathrm{m}$. Given this maximum grain temperature, we adopt an inner disk radius of $12 R_{*}$. The relatively low flux at $24 \mu \mathrm{m}$ means that the disk cannot extend too far outward, for otherwise there would be more cool dust and therefore more emission at this wavelength than measured. We therefore assume that the opaque disk is truncated at $50 R_{*}$. To fit the entire infrared continuum, we adopt a transition between region I and region II at $30 R_{*}$, although the overall fit is insensitive to this choice and region I could extend between $12 R_{*}$ and $50 R_{*}$. In order that the grains at the top of the disk in region II not exceed $1200 \mathrm{~K}$, we adopt $\alpha=1.5$, which is achieved by our model glassy grains with radii of $2 \mu \mathrm{m}$.

In our model, the silicate emission is largely produced in region III, the optically thin outer portion of the disk beyond $50 R_{*}$. So that the grain temperature lies below about $1200 \mathrm{~K}$, we adopt $\alpha=5$, which is characteristic of our model glassy grains with a radius of $1 \mu \mathrm{m}$. To enable the very strong silicate feature to reprocess about $1 \%$ of the total luminosity of the star, we assume region III is warped because a flat disk cannot intercept enough light from the illuminating star. The maximum fraction of the star's luminosity that a flat disk of inner radius $R$ can re-emit is $\left(4 R_{*}\right) /(3 \pi R)$, and with $R=50 R_{*}$, the outer portion of the disk can reprocess at most $1 \%$ of the energy of the star. Since the dust might emit only $\sim 50 \%$ of its light in the silicate feature, the outer portion of a flat disk cannot account for the strength of the feature. There is a degeneracy between the warp angle and the outer radius of region III of the disk. Since the grains that produce the silicate emission cannot also produce much emission at $24 \mu \mathrm{m}$, we infer that these particles are relatively warm. We therefore adopt $70 R_{*}$ as the outer radius of the warped thin disk. With this outer radius, the strength of the silicate feature is reproduced with a mean warp angle of $7.5^{\circ}$.
We display in Figure 3 a comparison between the data and the reasonably fitting model. We somewhat overestimate the flux at $24 \mu \mathrm{m}$, but this measurement is only a $4 \sigma$ result and therefore somewhat uncertain. Also, the width of the peak in the model spectrum is somewhat narrower than that shown by the data, so other materials besides glassy silicates might be present in the disk. Crystalline material may contribute to the shape of the silicate emission, and a spectrum longward of $15 \mu \mathrm{m}$ can test this possibility (Jaeger et al. 1998). Regardless of these uncertainties, it seems that the dust lies fully within the star's tidal radius of $\sim 1 R_{\odot}$, consistent with the hypothesis that the dust disk around GD 362 was created by tidal disruption of an asteroidal-size parent body.

\section{DISCUSSION}

Our results constrain possible models of the origin of the dust near GD 362. For optically thin emission, the mass of circumstellar dust, $M_{\text {dust }}$, is

$$
M_{\text {dust }}=\frac{F_{\nu} D_{*}^{2}}{\chi_{\nu} B_{\nu}(T)},
$$

where $F_{\nu}$ is the flux. Consider the silicate feature. The peak flux is about $1.5 \mathrm{mJy}$ and with $T=1000 \mathrm{~K}$ (see Table 2) and $\chi_{\nu}=$ $3000 \mathrm{~cm}^{2} \mathrm{~g}^{-1}$ (Dorschner et al. 1995), $M_{\text {dust }} \sim 3 \times 10^{17} \mathrm{~g}$ if the distance is $25 \mathrm{pc}$ as assumed by Becklin et al. (2005). Since it is likely that the star is farther away than previously assumed, the mass in dust is likely to be at least $10^{18} \mathrm{~g}$. If, however, the atmosphere has a convective envelope characteristic of a heliumrich white dwarf at 10,000 $\mathrm{K}$ of $\sim 1 M_{\oplus}$ (MacDonald et al. 1998), and if $0.1 \%$ of this mass is composed of metals (Gianninas et al. 2004), then the star may have already accreted more than $10^{24} \mathrm{~g}$. Consequently, it is plausible that there is much more circumstellar mass than inferred from the silicate emission feature. Supported by our modeling of the infrared spectral energy distribution, we suggest that the bulk of the circumstellar mass resides in an opaque, flat disk.

Garcia-Berro et al. (2007) proposed that GD 362 is the result of the merger of two lower mass white dwarfs and that the circumstellar disk is a remnant of this event (see also Livio et al. 2005). One motivation for this model was that, according to Gianninas et al. (2004), the mass of GD 362 is $1.24 M_{\odot}$. However, with the detection of helium in the photosphere, the gravity and mass of this star are smaller than previously derived and it seems much less likely that GD 362 is the product of a merger. In any case, even young massive white dwarfs do not characteristically display infrared excesses from dust (Hansen et al. 2006). A test of the merger model for GD 362 is to measure the composition of the star's atmosphere (B. Zuckerman et al. 2007, in preparation) to compare with the nucleosynthetic predictions for such an event.

The relatively weak $24 \mu \mathrm{m}$ flux is best understood if there is little cold dust in the system. Interstellar accretion (Koester \& Wilken 2006) results in matter drifting radially inward from the Bondi-Hoyle accretion radius, which is typically greater than 1 AU (see Spitzer 1978). In our model, the dust is all located within $0.01 \mathrm{AU}$. A similar argument applies to the dust orbiting G29-38, for which Reach et al. (2005a) measured $F_{\nu}(24 \mu \mathrm{m})=$ $2.4 \mathrm{mJy}$. If the opaque, geometrically flat disk described by Jura (2003) extends to much more than approximately $1 R_{\odot}$, then the predicted flux is near $16 \mathrm{mJy}$. We therefore interpret the data for G29-38 as evidence that the disk around this star has an outer truncation within $1 R_{\odot}$. It is possible that there is cold dust much farther from the star that is unrecognizable with current data. 
The model that the dust is located in an orbiting disk is dynamically plausible and is consistent with the infrared observations. However, we do not have any direct measurements of the disk dynamics or geometry. An indirect measurement that constrains the dust geometry in G29-38, a white dwarf that bears many similarities to GD 362, is given by the periodic intensity variations in the infrared disk emission that are driven by the nonradial opticallight variations of this ZZ Ceti class star (Graham et al. 1990; Patterson et al. 1991). These timing data exclude the possibility, raised by the optically thin model of Reach et al. (2005a), that G29-38's circumstellar dust is distributed in a spherical shell. If GD 362 is variable (see Gianninas et al. 2006 for a recent discussion of ZZ Ceti class stars), then the spatial distribution of the circumstellar dust could be probed by measuring time variations of the infrared excess.

It is possible that the mass in dust orbiting GD 362 substantially exceeds $10^{20} \mathrm{~g}$ and therefore that the diameter of the tidally distorted parent body was considerably larger than $30 \mathrm{~km}$. With available data, we cannot determine whether this parent body was more analogous to an asteroid or to a Kuiper Belt object. Jura (2006) has argued that values of $n(\mathrm{C}) / n(\mathrm{Fe})$ in three externally polluted helium-rich white dwarfs are at least a factor of 10 below solar, strongly favoring accretion of asteroid-like bodies with a chondritic composition. Measurements of the carbon abundances in the atmospheres of G29-38 and GD 362 may strongly constrain the nature of the parent body pollution in these and other metalenriched white dwarf atmospheres (Zuckerman et al. 2003).

\section{CONCLUSIONS}

We report Spitzer Space Telescope observations of GD 362. We approximately reproduce both the infrared continuum and the strong silicate emission with a model consistent with the hypothesis that the dusty disk around GD 362 is the debris from a tidally disrupted asteroidal-size body. The weak $24 \mu \mathrm{m}$ flux argues against much cold dust being present in the system as would be expected if interstellar accretion is important. Determination of the metal abundance within the atmosphere of this star (B. Zuckerman et al. 2007, in preparation) may indirectly measure the bulk composition of an extrasolar minor planet.

This work has been partly supported by NASA.

\section{APPENDIX}

Following Pringle (1991), we present a simple description of a dust disk to assess whether it can be warped. We assume that the disk is formed from an asteroidal-size body that is initially disrupted at its periastron, $R_{\text {per }}$, and relatively quickly forms a thin ring that then expands under the action of its own viscosity to form an orbiting disk with mass $M_{\text {disk. }}$. After the initial conditions damp out, the surface density $\Sigma$ is conveniently expressed (Jura et al. 2002) as

$$
\Sigma=\frac{M_{\text {disk }}}{\left(12 \pi^{3} R^{3} v_{\text {vis }} t\right)^{1 / 2}} \exp \left(-\frac{R}{3 v_{\text {vis }} t}\right),
$$

where $v_{\text {vis }}$ is the radial flow speed due to viscosity and $t$ is the age of the disk. We define $\epsilon$ as

$$
\epsilon=\frac{v_{\text {vis }} t}{R_{\text {per }}},
$$

so that $\epsilon$ measures the ratio of the spreading distance of the disk compared to its initial location. Because the value of $v_{\text {vis }}$ is not well measured, we use $\epsilon$ to characterize a mature disk. If $\epsilon \ll 1$, then the disk has not had much time to evolve from its initial configuration, while if $\epsilon \gg 1$, the disk may have largely dissipated. Therefore, we adopt $\epsilon \sim 1$ for the systems of interest.

While a warp might be driven by hydromagnetic (Quillen 2001) or magnetic (Lai 1999) torques, we consider radiatively driven warping. According to Pringle (1996), if the disk is opaque in the infrared, it is unstable to self-induced warping if the star's luminosity $L_{*}$ is large enough that the back-reaction of radiation leaving the surface of the disk can induce a sufficiently strong torque, which occurs if

$$
L_{*}>12 \pi^{2} \nu_{2}\left(\frac{G M_{*}}{R}\right)^{1 / 2} \Sigma,
$$

where $\nu_{2}$ is the azimuthal viscosity. Making the uncertain extrapolation that the azimuthal and radial viscosities are equal, we write $\nu_{2}=R v_{\text {vis }}$, and then using equation (A1), warping occurs where

$$
R>\frac{\left(12 \pi \epsilon R_{\mathrm{per}} G M_{*}\right)^{1 / 2}}{L_{*} t} c M_{\text {disk }} \exp \left(-\frac{R}{3 \epsilon R_{\mathrm{per}}}\right) .
$$

For GD 362 the relevant parameters are very uncertain. For illustrative purposes, we adopt $L_{*}=2 \times 10^{-3} L_{\odot}, M_{*}=0.8 M_{\odot}$, and $M_{\text {disk }}=10^{24} \mathrm{~g}$. Given that the accretion rate might be $\sim 10^{11} \mathrm{~g} \mathrm{~s}^{-1}$ (Becklin et al. 2005), we estimate $t=10^{5} \mathrm{yr}, \epsilon=1$, and $R_{\text {per }}=3 \times 10^{10} \mathrm{~cm}$. In this case, the disk is warped for $R$ larger than $\sim 50 R_{*}$.

The disk is unstable to radiative warping only where it is optically thick. We assume that such warping occurs, and that the disk then expands further and becomes optically thin. In this very outermost region, the decay time for the warp can be as long as the disk expansion time (Armitage \& Pringle 1997), and therefore the optically thin portion of the disk effectively retains the warp it acquired when it was more compact. 


\section{REFERENCES}

Armitage, P. J., \& Pringle, J. E. 1997, ApJ, 488, L47

Becklin, E. E., Farihi, J., Jura, M., Song, I., Weinberger, A., \& Zuckerman, B. 2005, ApJ, 632, L119

Burke, J. R., \& Hollenbach, D. J. 1983, ApJ, 265, 223

Calvet, N., Patino, A., Magris, G., \& D'Alessio, P. 1991, ApJ, 380, 617

Chen, C. H., et al. 2006, ApJS, 166, 351

Chiang, E. I., \& Goldreich, P. 1997, ApJ, 490, 368

Dorschner, J., Begemann, B., Henning, Th., Jager, C., \& Mutschke, H. 1995, A\&A, 300, 503

Ebel, D. S., \& Grossman, L. 2000, Geochim. Cosmochim. Acta, 64, 339

Fazio, G., et al. 2004, ApJS, 154, 10

Garcia-Berro, E., et al. 2007, in ASP Conf. Ser., EUROWD06: 15th European White Dwarf Workshop, ed. R. Napiwotzki \& M. Barstow (San Francisco: ASP), in press

Gianninas, A., Bergeron, P., \& Fontaine, G. 2006, AJ, 132, 831

Gianninas, A., Dufour, P., \& Bergeron, P. 2004, ApJ, 617, L57

Goody, R. M., \& Yung, Y. L. 1989, Atmospheric Radiation: Theoretical Basis (2nd ed.; New York: Oxford Univ. Press)

Graham, J. R., Matthews, K., Neugebauer, G., \& Soifer, B. T. 1990, ApJ, 357, 216

Hansen, B. M. S., Kulkarni, S., \& Wiktorowicz, S. 2006, AJ, 131, 1106

Hines, D., et al. 2006, ApJ, 638, 1070

Houck, J., et al. 2004, ApJS, 154, 18

Jaeger, C., Molster, F. J., Dorschner, J., Henning, Th., Mutschke, H., \& Waters, L. B. F. M. 1998, A\&A, 339, 904

Jura, M. 2003, ApJ, 584, L91

. 2006, ApJ, 653, 613

Jura, M., Chen, C., \& Plavchan, P. 2002, ApJ, 574, 963

Kemper, F., Vriend, W. J., \& Tielens, A. G. G. M. 2004, ApJ, 609, 826

Kilic, M., Mendez, R. A., von Hippel, T., \& Winget, D. E. 2005, ApJ, 632, L115
Kilic, M., von Hippel, T., Mullally, F., Reach, W. T., Kuchner, M. J., Winget, D. E., \& Burrows, A. 2006, ApJ, 642, 1051

Koester, D., \& Wilkin, D. 2006, A\&A, 453, 1051

Lai, D. 1999, ApJ, 524, 1030

Livio, M., Pringle, J. E., \& Wood, K. 2005, ApJ, 632, L37

MacDonald, J., Hernanz, M., \& Jose, J. 1998, MNRAS, 296, 523

McCord, T. B., \& Sotin, C. 2005, J. Geophys. Res., 110, 5009

Paquette, C., Pelletier, C., Fontaine, G., \& Michaud, G. 1986, ApJS, 61, 197

Patterson, J., Zuckerman, B., Becklin, E. E., Tholen, D. J., \& Hawarden, T. 1991, ApJ, 374, 330

Pringle, J. E. 1991, MNRAS, 248, 754 . 1996, MNRAS, 281, 357

Quijada, M. A., Marx, C. T., Arendt, R. G., \& Moseley, S. H. 2004, Proc. SPIE, 5487, 244

Quillen, A. 2001, ApJ, 563, 313

Reach, W. T., Kuchner, M., von Hippel, T., Burrows, A., Mullally, F., Kilic, M., \& Winget, D. E. 2005a, ApJ, 635, L161

Reach, W. T., et al. 2005b, PASP, 117, 978

Rieke, G., et al. 2004, ApJS, 154, 25

Song, I., Zuckerman, B., Weinberger, A. J., \& Becklin, E. E. 2005, Nature, 436, 363

Spitzer, L. 1978, Physical Processes in the Interstellar Medium (New York: Wiley)

Thomas, P. C., Parker, J. Wm., McFadden, L. A., Russell, C. T., Stern, S. A., Sykes, M. V., \& Young, E. F. 2005, Nature, 437, 224

Tremblay, P.-E., \& Bergeron, P. 2007, in ASP Conf. Ser., EUROWD06: 15th European White Dwarf Workshop, ed. R. Napiwotzki \& M. Barstow (San Francisco: ASP), in press

Werner, M. W., et al. 2004, ApJS, 154, 1

Zuckerman, B., \& Becklin, E. E. 1987, Nature, 330, 138

Zuckerman, B., Koester, D., Reid, I. N., \& Hunsch, M. 2003, ApJ, 596, 477 